\title{
Adjuvant Intraluminal Therapies in Upper Urinary Tract Urothelial Tumors
}

\author{
(1) Musab Ali Kutluhan ${ }^{1}$, (1) Ayhan Verit ${ }^{2}$ \\ ${ }^{1}$ Ankara Yıldırım Beyazıt University Faculty of Medicine, Department of Urology, Ankara, Turkey \\ 2University of Health Sciences Turkey, Fatih Sultan Mehmet Training and Research Hospital, Clinic of Urology, Istanbul, Turkey
}

\begin{abstract}
The gold standard treatment option for upper urinary tract urethelial tumors is radical nephroureterectomy and bladder cuff resection. On the other hand, with the development of surgical techniques, improvement in endoscopic instruments and better risk stratification in recent years, minimally invasive endoscopic procedures and renal-sparing surgical approaches such as segmental/total ureterectomy have begun to be applied. Metachronous tumor recurrences can be seen despite the use of renal-sparing approaches in selected patients. Adjuvant intraluminal treatments are applied in upper urinary tract tumors to reduce tumor recurrences. In this review, we discussed the role of adjuvant intraluminal therapies.
\end{abstract}

Keywords: Upper urinary tract urethelial tumor, renal-sparing approach, adjuvant intraluminal therapy

\section{Introduction}

Urothelial tumors take the $4^{\text {th }}$ place among cancers seen in developed countries (1). Upper urinary tract urothelial tumors (UUTUTs) are relatively rare and constitute $5-10 \%$ of all urothelial tumors (2). Although it is mostly diagnosed unilaterally, synchronous bilateral UUTUTs can be seen rarely (1.6\%) (3). The incidence of contralateral UUTUTs has been reported as $1-6 \%$ in the literature (4). It is thought that environmental factors may be effective in the formation of UUTUTs. Smoking and aristolochic acid are scientifically proven environmental factors in etiology $(5,6)$. In addition, there are strong findings that there is a relationship between hereditary UUTUTs and hereditary non-polyposis colorectal carcinoma (7).

The gold standard treatment option in UUTUTs is radical nephroureterectomy and resection around the bladder orifice (8). However, in recent years, the view that radical nephroureterectomy may be overtreatment in low-stage and high-risk UUTUTs has been accepted (9). On the other hand, the development in surgical techniques and endoscopic instruments used in recent years allows better risk stratification and the introduction of minimally invasive endoscopic procedures such as segmental/total ureterectomy and renalsparing surgical approaches $(10,11)$. On the other hand, metachronous tumor recurrences can be seen despite the use of renal-sparing approaches in selected patients. Adjuvant intraluminal treatments are applied in UUTUTs to reduce tumor recurrences. In this review, we discussed the role of adjuvant intraluminal therapies.

\begin{abstract}
Renal-sparing Surgery Indications and Risk Stratification
European Urology Guidelines defined indications for renalsparing surgery (12). Anatomical or functional solitary kidney, presence of bilateral tumors, detection of kidney failure, and poor performance of the patient for radical surgery are definite indications. However, low-risk tumor or selected patients with high-risk tumors (multifocal tumors, large, low-risk and superficial tumors) are relative indications (Table 1). In addition, the risk stratification of UUTUTs was defined in the 2019 European Urology Guideline to determine which patients were more suitable for a renal-sparing approach (12). According to the European Urology Guideline risk stratification; single focus, tumor size $<2 \mathrm{~cm}$, low-grade cytology or ureteroscopic biopsy results, and no invasive spread on computer tomographyurography are features of low-risk UUTUT. Presence of hydronephrosis, tumor size $>2 \mathrm{~cm}$, high-grade cytology or ureteroscopic biopsy result, multifocal tumor, variant histology, and a history of radical cystectomy are features of a high-risk UUTUT (Table 2).
\end{abstract}

\section{Renal-sparing Endoscopic Approach}

In recent years, with the development of endoscopic instruments, retrograde or antegrade endoscopic approach has been started

Cite this article as: Kutluhan MA, Verit A. Adjuvant Intraluminal Therapies in Upper Urinary Tract Urothelial Tumors. Bull Urooncol 2021;20(3):129-132

Address for Correspondence: Musab Ali Kutluhan, Ankara Yıldırım Beyazıt University Faculty of Medicine, Department of Urology, Ankara, Turkey Phone: +90 2165783000 E-mail: dr.musab151@gmail.com ORCID-ID: orcid.org/0000-0001-7117-9210 Received: 25.09.2019 Accepted: 31.10.2020 
Table 1. Indications for renal-sparing surgery in upper urinary tract urothelial tumors

\section{Strong Recommendations}

Anatomical or functional solitary kidney

Bilateral tumor

Chronic renal failure

Not suitable for radical nephroureterectomy

Weak Recommendations

Low risk upper urinary tract urothelial tumor

Appropriate high-risk upper urinary tract urothelial tumor: Multifocal

tumor, large tumor, tumor with low-risk features, superficial tumor

Table 2. Risk stratification in upper urinary tract urothelial tumors

High risk upper urinary tract urothelial tumor

- Hydronephrosis

- High grade (Ureteroscopic biopsy or cytology)

- Tumor size $>2 \mathrm{~cm}$

- Multifocal disease

- Variant histology

- History of radical cystectomy

Low risk upper urinary tract urothelial tumor

- Single focus

- Tumor size $<2 \mathrm{~cm}$

- Low grade (Ureteroscopic biopsy or cytology)

- No invasive spread in computer tomography-urography

to be used frequently in UUTUTs, especially in low-risk patient group. In a meta-analysis, Seisen T. showed that low-risk patients who underwent ureteroscopy or percutaneous renal-sparing surgical approach had similar survival compared to patients who underwent radical nephroureterectomy (13). Similar oncological outcomes, better renal function after renal-sparing surgery, and lower morbidity made the renal-sparing approach to be preferred more often than the radical approach in lowrisk patients. The current European Urology Guideline states that endoscopic ablation can be applied in low-risk patients if there are suitable instruments for ablation and biopsy, if there is a flexible ureteroscope, if the patient is informed about the need for close follow-up, and if complete tumor resection can be performed (14). Although the percutaneous approach is applied in patients with low-risk renal pelvis or calyx tumors, it has been used less frequently in recent years due to the development of retrograde endoscopic instruments and the risk of tumor seeding in the percutaneous approach (15).

\section{Intraluminal Treatments}

Although the indications for the use of intraluminal therapies in bladder cancer are stated in European and American Urology Guidelines, there is still insufficient evidence regarding the use of intraluminal therapies in UUTUTs. Although there are no randomized clinical studies conducted to date, most of the available data in the literature are based on retrospective studies.

Intraluminal treatments are applied antegrade or retrograde way in UUTUTs. In both methods, there is no standard approach in terms of duration, frequency, and the agent applied. For antegrade administration, a 10F percutaneous nephrostomy tube is inserted into the patient and it is waited for 2 weeks for the tract to form before starting the infusion. For antegrade intraluminal immunotherapy [Bacillus Calmette-Guerin (BCG)], $360 \mathrm{mg}$ Immune BCG Pasteur or $243 \mathrm{mg}$ ImmuCyst is dissolved in $150 \mathrm{~mL} 0.9 \%$ saline and given as an infusion over 2 hours at $1 \mathrm{~mL}$ per minute over $20 \mathrm{~cm}$ above the patient's kidney level $(16,17)$. Antegrade intraluminal chemotherapy (mitomycin C) is administered using a similar procedure by dissolving $40 \mathrm{mg}$ of mitomycin in $20 \mathrm{~mL}$ of $0.9 \%$ saline. During the treatment, the nephrostomy tube is changed every 3 months (17). Although antegrade method with percutaneous nephrostomy tube is a more direct method, the nephrostomy tube should remain on the patient during the treatment process. This may adversely affect the patient's quality of life. On the other hand, in retrograde method, a 5F ureteral catheter is placed in the patient with cystoscopy. In patients who are infused through a ureteral catheter, the pressure of the manometer is kept below 20-30 $\mathrm{mmHg}$, and the patient is told to change positions every 15-20 minutes for the infusion to affect the entire urinary tract (right side, left side, supine and prone positions) (18). The agent used, dose and duration of administration are similar to antegrade method. Retrograde method is seen as a more comfortable method by patients, especially since it can be performed under local anesthesia and there is no permanent catheter on the patient during the treatment process. Before both applications, urine cultures are taken from the patients and prophylactic antibiotics are given. The induction dose for intraluminal chemotherapy (mitomycin C) and immunotherapy (BCG) is given once a week for 6 weeks. Although the maintenance dose for intraluminal chemotherapy is not clear, it is given once a month for at least 3 months (18). The maintenance dose for intraluminal BCG is once a week for 3 weeks at the $3^{\text {rd }}, 6^{\text {th }}, 12^{\text {th }}$, $18^{\text {th }}, 24^{\text {th }}, 30^{\text {th }}$ and $36^{\text {th }}$ months (19). One of the main problems in retrograde method is the time it takes for the applied agent to pass and act in the upper urinary tract tract. It should be ensured that the proximal end of the inserted catheter is in the renal pelvis and that there is no mucosal injury. Methods such as ureteral meatotomy and permanent ureteral stenting which causes retrograde reflux have been described. However, before induction therapy, it should be ensured that there is sufficient volume to activate the reflux (20).

It has been thought that intraluminal treatments would prevent recurrence after a renal-sparing approach, especially in low-risk patients, as in bladder cancer. Chemotherapeutic and immunotherapeutic agents have been applied for a short time in UUTUTs $(21,22)$. The results of adjuvant intraluminal BCG administration after UUTUT resection or ablation are not promising. In a study by Giannarini et al. (16), recurrence-free survival and progression-free survival (PFS) were found $41 \%$ and 59\%, respectively, in patients with Ta or T1 UUTUT treated with BCG antegrade perfusion as an induction regimen (16). In another study by Rastinehad et al. (23), no statistically significant difference was found in terms of recurrence in patients who received adjuvant intraluminal BCG in both high-grade and lowgrade UUTUTs compared to those who did not (low grade 26\%$33 \%$ vs high grade $38 \%-39 \%$ ). On the other hand, it has been shown that adjuvant intraluminal BCG induction therapy gives better results in upper urinary tract carcinoma in situ (CIS) (24). Carmignani et al. (25) evaluated 12 studies involving a total of 185 patients and stated that the mean recurrence rate was $32 \%$ 
in a mean follow-up period of 19-57 months after 6 weeks of BCG induction therapy in 165 patients with upper urinary tract CIS. On the other hand, Shapiro et al. (26) evaluated the role of BCG-interferon therapy in biopsy-confirmed upper urinary tract CIS in a study they conducted. In a study involving 11 patients, complete response was observed in 8 (73\%) patients and only 1 patient had biopsy-proven recurrence. Based on the available data in the literature, it can be concluded that adjuvant intraluminal BCG therapy is effective and can be used as a primary treatment option in upper urinary tract CIS without papillary tumor. However, prospective randomized clinical studies with larger patient populations are needed.

Just as adjuvant chemotherapeutic agents are used to prevent progression and recurrence after transurethral resection in bladder tumors; adjuvant chemotherapeutic agents are used to reduce recurrence after resection in low-risk patients with UUTUTs. The recurrence rate in patients who have received adjuvant intraluminal mitomycin c after resection is between $29-54 \%$, and the rate of nephroureterectomy is between $5-21 \%(27,28)$. In a study by Metcalfe et al. (18), 28 patients with low-grade Ta-T1 UUTUTs were given adjuvant induction and maintenance intraluminal mitomycin c after resection, and the 3-year recurrence-free, progression-free, and nephroureterectomy-free survival rates were $60 \%$, [ confidence interval $(\mathrm{Cl})(95 \% \mathrm{Cl}): 42,86 \%$ ], $80 \%(95 \% \mathrm{Cl}$ : $64,100 \%)$, and $76 \%(95 \% \mathrm{Cl}: 60,97 \%)$, respectively $(18)$. On the other hand, postoperative single dose intravesical chemotherapy can be applied to prevent bladder recurrence after radical nephroureterectomy. The bladder recurrence rate after radical nephroureterectomy in UUTUTs is $22-47 \%$. In a study by Alma et al. (29), bladder tumor recurrence was observed in $22.7 \%$ (5 patients) of 22 patients who underwent radical nephroureterectomy due to UUTUT during a mean follow-up period of 32 months. In a meta-analysis, it was shown that administration of a single dose of intravesical chemotherapy (mitomycin c, epuribicin) within 2-10 days after radical nephroureterectomy statistically significantly reduced the rate of bladder recurrence within 1 year (30). In the current European Urology Guideline, post-operative singledose intravesical chemotherapy is recommended after radical nephroureterectomy.

\section{Conclusion}

Although minimally invasive renal-sparing surgical methods have been used frequently with the development of endoscopic instruments in low-risk patients with UUTUTs, the role of adjuvant intraluminal therapies used to reduce recurrence and progression after renal-sparing surgery is still not clear according to current literature data. There are still no recommendations regarding adjuvant intraluminal therapies in the current European Urology Guidelines. Randomized clinical trials with larger populations and meta-analyses investigating the effectiveness of adjuvant intraluminal therapies in UUTUTs are needed.

\section{Acknowledgements}

Publication: The results of the study were not published in full or in part in form of abstracts.

Contribution: There is not any contributors who may not be listed as authors.
Conflict of Interest: No conflict of interest was declared by the authors.

Financial Disclosure: The authors declared that this study received no financial support.

Ethics

Peer-review: Externally peer-reviewed.

\section{Authorship Contributions}

Concept: M.A.K., A.V., Design: M.A.K., A.V., Data Collection and Processing: M.A.K., A.V., Analysis and Interpretation: M.A.K., A.V., Literature Search: M.A.K., A.V., Writing: M.A.K., A.V.

\section{References}

1. Siegel RL, Miller KD, Jemal A. Cancer statistics CA Cancer J Clin 2017;67:7-30.

2. Siegel RL, Miller KD, Jemal A. Cancer statistics, CA Cancer J Clin 2015;65:5-29.

3. Holmäng S, Johansson SL. Synchronous bilateral ureteral and renal pelvic carcinomas: incidence, etiology, treatment and outcome. Cancer 2004;101:741-747.

4. Novara G, De Marco V, Dalpiaz O, et al. Independent predictors of contralateral metachronous upper urinary tract transitional cell carcinoma after nephroureterectomy: multi-institutional dataset from three European centers. Int J Urol 2009;16:187-191.

5. Guillaume L, Guy L. Epidemiology of and risk Factors for bladder cancer and for urothelial tumors. Rev Prat 2014;64:1372-1374.

6. Grollman AP. Aristolochic acid nephropathy: Harbinger of a global iatrogenic disease. Environ Mol Mutagen 2013;54:1-7.

7. Roupret $M$, Yates DR, Comperat $E$, et al. Upper urinary tract urothelial cell carcinomas and other urological malignancies involved in the hereditary non polyposis colorectal cancer (lynch syndrome) tumor spectrum. Eur Urol 2008;54:1226-1236.

8. Margulis, V, Shariat SF, Matin SF, et al. Outcomes of radical nephroureterectomy: a series from the Upper Tract Urothelial Carcinoma Collaboration. Cancer 2009;115:1224-1233.

9. Clark PE, Streem SB, Geisinger MA. 13-year experience with percutaneous management of upper tract transitional cell carcinoma. J Urol 1999;161:772-775.

10. Fang D, Seisen T, Yang K, et al. A systematic review and meta-analysis of oncological and renal function outcomes obtained after segmental ureterectomy versus radical nephroureterectomy for upper tract urothelial carcinoma. Eur J Surg Oncol 2016;42:1625-1635.

11. Grasso M, Fishman Al, Cohen J, et al. Ureteroscopic and extirpative treatment of upper urinary tract urothelial carcinoma: a 15year comprehensive review of 160 consecutive patients. BJU Int 2012;110:1618-1626.

12. Rouprêt $M$, Babjuk $M$, Burger $M$, et al. European Association Of Urology Guidelines on upper urinary tract urothelial cell carcinoma: Prognosis. Eur Urol 2019:79:62-79.

13. Seisen T, Peyronnet B, Dominguez-Escrig JL, et al. Oncologic outcomes of kidney-sparing surgery versus radical nephroureterectomy for upper tract urothelial carcinoma: a systematic review by the EAU Non muscle Invasive Bladder Cancer Guidelines panel. Eur Urol 2016;70:1052-1068.

14. Rouprêt $M$, Babjuk $M$, Burger $M$. et al. European Association of Urology Guidelines on upper urinary tract urothelial cell carcinoma: disease management. Eur Urol 2019:79:62-79.

15. Roupret $\mathrm{M}$, Traxer $\mathrm{O}$, Tligui $\mathrm{M}$, et al. Upper urinary tract transitional cell carcinoma: recurrence rate after percutaneous endoscopic resection. Eur Urol 2007;51:709-713.

16. Giannarini G, Kessler TM, Birkhauser FD, et al. Antegrade perfusion with bacillus Calmette Guerin in patients with non-muscle-invasive 
urothelial carcinoma of the upper urinary tract: Who may benefit? Eur Urol 2011;60:955-960.

17. Balasubramanian A, Metcalfe M, Wagenheim G, et al. Salvage topical therapy for upper tract urothelial carcinoma. World J Urol 2018;36:2027-2034.

18. Metcalfe M, Wagenheim G, Xiao L, et al. Induction and maintenance adjuvant mitomycin $C$ topical therapy for upper tract urothelial carcinoma: tolerability and intermediate term outcomes. J Endourol 2017;31:946-953.

19. Lamm DL. Preventing progression and improving survival with BCG maintenance. Eur Urol 2000;37(Suppl 1):9-15.

20. Yossepowitch O, Lifshitz DA, Dekel, Y, et al. Assessment of vesicoureteral reflux in patients with self-retaining ureteral stents: implications for upper urinary tract instillation. J Urol 2005;173:890-893.

21. Vasavada SP, Streem SB, Novick AC. Definitive tumor resection and percutaneous bacille Calmette-Guérin for management of renal pelvic transitional cell carcinoma in solitary kidneys. Urology 1995;45:381-386.

22. Keeley FX Jr, Bagley DH. Adjuvant mitomycin C following endoscopic treatment of upper tract transitional cell carcinoma. J Urol 1997; 158:2074-2077.

23. Rastinehad AR, Ost MC, Vanderbrink BA, et al. A 20-year experience with percutaneous resection of upper tract transitional carcinoma: is there an oncologic benefit with adjuvant bacillus Calmette Guerin therapy? Urology 2009;73:27-31.

24. Cutress ML, Stewart GD, Zakikhani P, et al. Ureteroscopic and percutaneous management of upper tract urothelial carcinoma (UTUC): systematic review. BJU Int 2012;110:614-628.

25. Carmignani L, Bianchi R, Cozzi G, et al. Intracavitary immunotherapy and chemotherapy for upper urinary tract cancer: current evidence. Rev Urol 2013;15:145-153.

26. Shapiro EY, Lipsky MJ, Cha DY, et al. Outcomes of intra renal Bacillus Calmette Guerin/interferon- alpha2B for biopsy-proven upper-tract carcinoma in situ. J Endourol 2012;26:1645-1650.

27. Aboumarzouk OM, Somani B, Ahmad S, et al. Mitomycin C instillation following ureterorenoscopic laser ablation of upper urinary tract carcinoma. Urol Ann 2013;5:184-189.

28. Wagenheim GN, Papadopoulos J, Navai N, et al. Mitomycin-c induction and maintenance topical therapy for upper tract urothelial carcinoma. J Endourol 2017;31:946-953.

29. Alma E, Erçil H, Eken A, et. al. Intravesical Recurrence After Nephroureterectomy in Patients with Upper Urinary Tract Urothelial Carcinoma J Reconstr Urol 2018;8:55-60.

30. Fang D, Li XS, Xiong GY, et al. Prophylactic intravesical chemotherapy to prevent bladder tumors after nephroureterectomy for primary upper urinary tract urothelial carcinomas: a systematic review and meta-analysis. Urol Int 2013;91:291-296. 